\title{
ASSOCIAÇÃO ENTRE A ANALGESIA EPIDURAL E O TRAUMA PERINEAL NO PARTO VAGINAL
}

\section{Association between epidural analgesia and perineal laceration in vaginal delivery}

\section{Asociación entre la analgesia epidural y el trauma perineal en el parto vaginal}

\author{
Juliana Cristina dos Santos Monteiro \\ Ana Carolina Sartorato Beleza ${ }^{4}$
}

\author{
Ana Carolina Rodarti Pitangui ${ }^{2}$ \\ Ana Márcia Spanó Nakano ${ }^{5}$
}

\author{
Ligia de Sousa ${ }^{3}$ \\ Flávia Azevedo Gomes ${ }^{6}$
}

\section{RESUMO}

. 0 objetivo deste estudo foi analisar a associação entre a analgesia epidural e a laceração perineal em mulheres submetidas ao parto vaginal. Foi realizado um estudo descritivo e transversal, para o qual foram pesquisados 109 prontuários de mulheres assistidas durante o processo de parturição, em uma maternidade em Ribeirão Preto. Os dados foram coletados nos meses de março e abril de 2003. Para analisar a correlação entre as variáveis foi aplicado o teste qui-quadrado. Foi realizado parto normal em $91,7 \%$ (100) das parturientes e fórceps em $8,2 \%$ (9). Na análise dos dados, $74,3 \%$ da amostra receberam analgesia epidural; destas, 26,5\% tiveram algum grau de laceração perineal e 9,1\%, períneo íntegro, não sendo possível verificar a associação entre as variáveis mencionadas. Não foi verificada, neste estudo, significância estatística para afirmar que as condições do períneo após o parto vaginal e a utilização da analgesia epidural estão associadas (valor x² $4 \mathrm{GL}=3,1$ ).

Palavras-chave: Parto Normal. Analgesia Epidural. Períneo. Saúde da Mulher.

\begin{abstract}
The aim of this study was to analyze the association between the epidural analgesia and the perineal laceration in women submitted to a vaginal childbirth. A descriptive and transversal study was realized with 109 archives of women attended during the childbirth process in a maternity located in Ribeirão Preto. The data was collected in March and April 2003. To analyze the correlation between the variables, the Chi-Square Test was applied. Was realized normal childbirth in $91,7 \%$ of the parturient and forceps labor in $8,2 \%$. In the data analysis, $74.3 \%$ of the sample received epidural analgesia, $26.5 \%$ had some degree of laceration perineal and $9.1 \%$ had intact perineum. The results demonstrated none association between the mentioned variables. No statistical significance was found in this study to affirm that the conditions of the perineum after the vaginal childbirth and the use of the epidural analgesia are associates $\left(x^{2} 4 G L=3,1\right)$.
\end{abstract}

Keywords: . Natural childbirth. Analgesia, Epidural. Perineum. Women's health.

\section{Resumen}

El objetivo de esto estudio fue analizar la asociación entre la analgesia epidural y la laceración perineal en mujeres sometidas al parto vaginal. Fue realizado un estudio descriptivo y transversal, para lo cual fueron investigados 109 prontuarios de mujeres asistidas durante el proceso de parturición en una maternidad en Ribeirão Preto. Los datos fueron colectados en marzo y abril de 2003. Para analizar la correlación entre las variables fue aplicado el Teste Chi-Cuadrado. Fue realizado parto normal en $91,7 \%$ (100) de las parturientes y en $8,2 \%$ (09) parto fórceps. En el análisis de los datos, 74,3\% de la muestra recibieron analgesia epidural, y de estas $26,5 \%$ tuvieron algún nivel de laceración perineal y $9,1 \%$ perineo íntegro. No fue posible verificar la asociación entre las variables aludidas. No fue verificado en esto estudio, significancia estadística para afirmar que las condiciones del perineo después del parto vaginal y el uso de analgesia epidural, están asociadas $\left(x^{2} 4 G L=3,1\right)$.

Palabras clave: Parto normal. Analgesia epidural. Períneo. Salud de la mujer.

'Enfermeira Obstétrica, Mestre em Enfermagem em Saúde Pública, Doutoranda no Programa de Pós-Graduação Enfermagem em Saúde Pública da Escola de Enfermagem de Ribeirão Preto da Universidade de São Paulo. Brasil. E-mail: jumonte@eerp.usp.br, ${ }^{2}$ Fisioterapeuta, Mestre em Enfermagem em Saúde Pública, Doutoranda no Programa de Pós-Graduação Enfermagem em Saúde Pública da Escola de Enfermagem de Ribeirão Preto da Universidade de São Paulo. Brasil. E-mail: carolpitangui@usp.br, ${ }^{3}$ Fisioterapeuta Mestre em Enfermagem em Saúde Pública, Doutoranda no Programa de Pós-Graduação Enfermagem em Saúde Pública da Escola de Enfermagem de Ribeirão Preto da Universidade de São Paulo. Brasil. E-mail: ligia.sousa@hotmail.com, ${ }^{4}$ Fisioterapeuta, Mestre em Enfermagem em Saúde Pública, Doutoranda no Programa de Pós-Graduação Enfermagem em Saúde Pública da Escola de Enfermagem de Ribeirão Preto da Universidade de São Paulo. Brasil. E-mail: acbeleza@terra.com.br, ${ }^{5}$ Enfermeira Obstétrica, Professora Associada do Departamento de Enfermagem Materno-Infantil e Saúde Pública da Escola de Enfermagem de Ribeirão Preto da Universidade de São Paulo. Brasil. E-mail: nakano@eerp.usp.br, ${ }^{6}$ Enfermeira Obstétrica, Professora Doutora do Departamento de Enfermagem Materno-Infantil e Saúde Pública da Escola de Enfermagem de Ribeirão Preto da Universidade de São Paulo. Brasil. E-mail: flagomes@uol.com.br 


\section{INTRODUCÃO}

0 trabalho de parto, apesar de ser um processo fisiológico, resulta em dor intensa para muitas mulheres ${ }^{1}$. Métodos não farmacológicos e farmacológicos podem auxiliar no controle da sensação dolorosa que acompanha este processo. Apesar de recomendações do uso de recursos não farmacológicos para alívio da dor, como massagens, hidroterapia e eletroestimulação nervosa transcutânea como parte da humanização do cuidado, muitos profissionais que assistem a parturiente indicam o uso de drogas anestésicas e analgésicas².

A analgesia epidural é um método moderno e popular na prática obstétrica, entretanto, não ausente de efeitos adversos. Sua eficácia no controle da dor durante o trabalho de parto é de grande valia, além do alivio considerável que traz para as mulheres que dela se utilizam. Pode ser ministrada em dose única, quando realizada próximo ao período expulsivo, em doses intermitentes ou em infusão contínua, dependendo da fase do trabalho de parto ${ }^{3}$. Outro recurso existente, que também é utilizado em alguns serviços de saúde, é a raquianestesia, bem como a sua combinação com a peridural.

Apesar dos benefícios mencionados pelo emprego da analgesia, atualmente, diversos estudiosos vêm investigando sua associação com a ocorrência do trauma perineal, pois esta prolongaria o período expulsivo, ocasionando uma maior utilização de intervenções obstétricas, que estariam relacionadas ao aumento no índice de lacerações. Porém, essas hipóteses são controversas, e muitas questões referentes à analgesia, traumas e sua influência na saúde e na experiência vivenciada pela parturiente ainda precisam ser esclarecidas ${ }^{4-5}$.

0 trauma perineal é descrito como sendo a perda da integridade do períneo ou qualquer outro dano ocorrido na região genital da mulher, podendo ser espontâneo ou decorrente de procedimento cirúrgico. As lesões cirúrgicas são aquelas causadas pelo uso da episiotomia, enquanto as espontâneas são as lacerações perineais que acometem a região genital da mulher, tanto mucosa como muscular. Estas apresentam diversos níveis de gravidade: laceração grau I envolve a pele e o tecido subcutâneo da região perineal, a mucosa vestibular e vaginal, porém não atinge fáscias nem músculos adjacentes; na laceração grau II, ocorre lesão perineal com ruptura posterior maior que três centímetros que envolve fáscias e músculos perineais, mas não o esfíncter anal; laceração grau III alcança o músculo retal, ocorrendo laceração parcial ou total do esfíncter anal; e laceração grau IV expõe a luz do reto, alcançando, além do músculo, a mucosa retal ${ }^{6-7}$.

Ultimamente, a literatura científica tem reportado este dano como sendo a maior causa de morbidade materna durante o parto vaginal, podendo ocasionar diversas consequências para a saúde da puérpera, como dor e desconfor tos que podem persistir tanto no pós-parto imediato quanto nos períodos mais distantes do puerpério ${ }^{4,8}$
Esses comprometimentos, além de acarretarem dor de alta intensidade e custos adicionais aos serviços de saúde, também trazem diversas limitações nas atividades de vida diária dessas mulheres, tais como: andar, sentar, dormir, evacuar, urinar, nos cuidados com o recém-nascido, na deficiência de lactação e na falta de sono e de apetite ${ }^{9}$.

Portanto, uma possível associação entre as variáveis analgesia epidural e trauma perineal seria razão para um redimensionamento das indicações deste recurso de alívio de dor pesando-se a relação custo-benefício para as mulheres.

Diante da relevância do tema e da escassez de pesquisas sobre o mesmo, objetivou-se, neste estudo, analisar a associação entre a analgesia epidural e a laceração perineal em uma maternidade em Ribeirão Preto - SP.

\section{METODOLOGIA}

Esta pesquisa constitui-se de um estudo descritivo e transversal, para o qual foram pesquisados todos os prontuários de mulheres assistidas durante o processo de parturição em uma maternidade em Ribeirão Preto - São Paulo, nos meses de março e abril de 2003. Esta maternidade é uma instituição filantrópica que beneficia exclusivamente mulheres conveniadas ao Sistema Único de Saúde (SUS), atendendo partos de baixo risco e privilegiando o parto vaginal.

A amostra estudada foi composta por 116 mulheres, porém 7 destas foram excluídas por não atenderem os critérios de inclusão estabelecidos para esta pesquisa: parto vaginal e primiparidade. Portanto, a amostra final estabelecida foi de 109 parturientes.

Para a obtenção dos dados, foi elaborado um instrumento que contemplava todas as informações que interessavam à investigação, tais como as variáveis sociodemográficas: idade, estado civil e grau de escolaridade, presença de laceração perineal, indicação de episiotomia, tipo de parto vaginal (normal ou fórceps) e emprego de analgesia obstétrica epidural e local.

0 projeto foi aprovado no Comitê de Ética e Pesquisa da Escola de Enfermagem de Ribeirão Preto - USP, em sua 44a Reunião Ordinária, constando no protocolo número 0284/2002, atendendo assim, a determinação da resolução 196/96 do Conselho Nacional de Saúde.

Após a coleta, os dados foram eletronicamente compilados em uma planilha, por meio do programa Microsoft Excel/2000. Para a análise estatística dos dados, utilizou-se o programa EPI-INFO, versão 6.04. Foi aplicado o teste qui-quadrado para analisar a correlação entre as variáveis estudadas. 0 nível de significância calculado foi pd" 0,5. 


\section{RESULTADOS}

Das 109 parturientes da amostra, 91,7\% (100) foram submetidas ao parto normal e 8,2\% (9) ao parto com fórceps.

Caracterizando a amostra em relação às variáveis sociodemográficas, verificamos que $56,8 \%$ (62) das parturientes, apresentavam idade entre 15 e 19 anos, $34,8 \%$ (38) entre 20 e 25 anos, 5,5\% (6) entre 26 e 30 anos, $1,8 \%$ (2) entre 31 e 35 anos e $0,9 \%$ (01) entre 10 e 14 anos.

Em relação ao estado civil, 44,9\% (49) das mulheres mantinham união consensual, $28,4 \%$ (31) eram solteiras, 22,9\% (25) eram casadas, e em 3,6\% (4) dos prontuários não constavam informações relacionadas ao estado civil.

Quanto ao grau de instrução, 39,4\% (43) das parturientes pesquisadas tinham apenas o ensino fundamental incompleto;
$28,4 \%$ (31) apresentaram ensino médio incompleto; $22,9 \%$ (25), ensino médio completo; 5,5\% (6), ensino fundamental completo; e apenas 0,9\% (1), ensino superior completo. Em 2,7\% (3) dos prontuários, não havia informação sobre o grau de instrução.

Na análise do emprego de analgesia durante o trabalho de parto, verificou-se que $34,8 \%$ das mulheres foram submetidas à anestesia epidural com $6 \mathrm{~cm}$ de dilatação cervical; 39,4\%, à anestesia epidural com $7 \mathrm{~cm}$ de dilatação cervical ou mais; $\mathrm{e}$ 25,6\%, à anestesia local. Quanto às condições do períneo após o parto vaginal, $51,3 \%$ das mulheres sofreram episiotomia, $38,5 \%$ delas apresentaram algum grau de laceração perineal e $10 \%$ das mulheres apresentaram períneo íntegro

(Tabela 1).

Tabela 1 - Avaliação das condições do períneo quanto ao tipo de anestesia durante o trabalho de parto (Ribeirão Preto, 2003).

\begin{tabular}{lccccc}
\hline \multicolumn{2}{c}{ Anestesia } & & \multicolumn{2}{c}{ Condições do períneo } \\
\hline $\begin{array}{l}\text { Epidural até } 6 \\
\text { dilatação }\end{array}$ & & Íntegro & Episiotomia & Laceração & Total \\
$\begin{array}{l}\text { Epidural com } 7 \mathrm{~cm} \text { de } \\
\text { dilatação cervical ou mais }\end{array}$ & $4(5,5 \%)$ & $19(17,4 \%)$ & $13(11,9 \%)$ & $38(34,8 \%)$ \\
Anestesia local & $1(0,9 \%)$ & $14(12,8 \%)$ & $13(11,9 \%)$ & $28(25,6 \%)$ \\
\hline Total & $11(10 \%)$ & $56(51,3 \%)$ & $42(38,5 \%)$ & $109(100 \%)$ \\
\hline
\end{tabular}

Ao se verificar a relação entre o tipo de analgesia durante o trabalho de parto e a integridade do períneo após o parto vaginal, utilizando o teste qui-quadrado (com quatro graus de liberdade), obteve-se o valor $x^{2} 4 G L=3,1$ ( $p d " 0,5$ ). Sendo assim, não houve evidência estatística para afirmar que as condições do períneo e o tipo de anestesia estão associados.

\section{DISCUSSÃO}

A analgesia peridural durante o trabalho de parto se mostra como um eficiente recurso para controlar a dor em diferentes fases do trabalho de parto, ocasionando ausência da dor e efeito prolongado, controle da intensidade e extensão do bloqueio, ausência de depressão respiratória materna e fetal, sendo ideal para situações de urgência, como cesárea e eclâmpsia. Neste estudo avaliou-se a ocorrência de trauma perineal em parturientes de parto vaginal espontâneo ou a fórceps, submetidas à analgesia epidural ou anestesia local. Para isso, foi realizada uma análise dos prontuários de mulheres atendidas durante o processo de parturição em uma maternidade em Ribeirão Preto.

Atualmente, alguns estudos têm associado uma maior frequência de trauma perineal ao uso de analgesia epidural em mulheres primíparas ${ }^{5-6,10-11}$. Este fato ocorreria em razão de este grupo apresentar uma relativa inelasticidade do canal de parto, que aumentaria a intensidade de dor e dificultaria a parturição, ocasionando, assim, uma maior utilização de analgesia epidural ${ }^{11}$.
Com objetivo de identificar as características das pacientes que apresentavam trauma perineal após parto vaginal, pesquisadores conduziram um estudo observacional com 3.409 mulheres e averiguaram que $27,6 \%$ dos partos vaginais ministraram analgesia epidural, sendo as pacientes que a utilizaram mais sucessíveis às lesões perineais ${ }^{12}$.

Estudo randomizado e controlado, realizado com 459 multíparas submetidas a parto vaginal verificou que $73,3 \%$ das mulheres que apresentaram trauma perineal de grau III ou IV receberam analgesia epidural ${ }^{7}$.

Em um estudo retrospectivo realizado com 2.759 pacientes, avaliou-se se a analgesia epidural seria um fator de risco independente ao trauma perineal grave. Para isso, foram analisadas mulheres que se submeteram ao parto vaginal com e sem o uso de analgesia, sendo constatado que $10,2 \%$ das puérperas que aplicaram o recurso apresentaram trauma grave comparadas com 5,2\% que não o receberam. Concluiu-se, portanto com este experimento, que a analgesia epidural estava associada ao aumento do trauma grave, sendo evidenciado 
também que esta aumentaria em três vezes o uso de instrumentação, sendo este um fator determinante para o risco de laceração $0^{10}$.

Estudo desenvolvido na Suécia com 2.283 parturientes submetidas ao parto vaginal encontrou um maior índice de lacerações em primíparas do que em multíparas (93\% versus $66,2 \%)$, sugerindo que estas sofrem lesões menores e menos graves. Também foi averiguado que os fatores de risco relacionados com o trauma perineal de grau II foram a nãovisualização total ou parcial do períneo, a longa duração do primeiro e do segundo estágio do trabalho de parto, a presença de edema perineal e o uso de vácuo extrator, ocitocina e analgesia epidural ${ }^{6}$.

Também com o objetivo de determinar se a analgesia epidural estaria associada a diferentes níveis de lesão perineal grave durante o parto vaginal, autores avaliaram 1.942 partos de baixo risco de primíparas e observaram que $70,9 \%$ destas mulheres receberam analgesia epidural, sendo que 16,1\% apresentaram laceração grave, comparadas com $9,7 \%$ que não foram submetidas à analgesia. Como resultado desta análise, confirmou-se que a analgesia epidural estava associada ao aumento no índice de trauma grave, além do fato de esta também estar relacionada ao aumento do uso de instrumentação e de episiotomia ${ }^{5}$. Outros autores ${ }^{13}$, também pesquisando esta associação, demonstraram que, com a analgesia peridural, a utilização de instrumentalização é mais provável, o que torna o risco para a ocorrência de lacerações três vezes maior.

Em nosso estudo, $74,3 \%$ da amostra foram submetidas à analgesia epidural; destas, 38,5\% sofreram episiotomia, e $26,5 \%$ tiveram algum grau de laceração perineal. Foi verificado períneo íntegro em 9,1\% dos casos. Portanto, não foi possível com esses dados verificar a associação entre as variáveis mencionadas.

Contudo, esses resultados divergem dos estudos supracitados e assinala-se com ele a possibilidade de que 0 grau de relaxamento da musculatura do assoalho pélvico proporcionado pela analgesia bem como a duração do segundo estágio do trabalho de parto sejam fatores que devam ser considerados importantes na determinação de maiores taxas de laceração perineal.

Em pesquisa multicêntrica ${ }^{11}$ realizada para determinar associação entre analgesia peridural e aumento do risco de lacerações durante o trabalho de parto vaginal, os autores não encontraram evidências de que esta técnica afete a integridade do canal do parto, mas observaram um prolongamento do segundo estágio de parto nos casos em que a analgesia foi utilizada.
Vale salientar que em nossa pesquisa não foi possível verificar o tempo médio de duração do segundo estágio de parto, nem parâmetros específicos relacionados à quantidade de analgésicos ministrados e os efeitos alcançados. Cabe também mencionar o fato de se ter encontrado uma dificuldade importante na coleta dos dados que se reverteu em limitação da pesquisa, já que houve insuficiência de informações relevantes nos prontuários utilizados, tais como: a altura e o peso do recém-nascido, peso da mãe, posição materna e fetal durante o parto, duração dos estágios do trabalho de parto e preparo e condição do períneo durante a gestação.

\section{CONCLUSÃO}

Diante do exposto, foi possível verificar neste estudo que não houve significância estatística para afirmar que as condições do períneo após o parto vaginal e a utilização da analgesia epidural estão associadas. Contudo, também foi observado que a literatura científica que repor ta esta temática ainda é escassa, e que mais trabalhos nesta área devem ser desenvolvidos e discutidos com o objetivo de elucidar esta possível associação.

Agradecemos ao CNPQ pelo financiamento concedido para o desenvolvimento da pesquisa.

\section{REFERÊNCIAS}

1 Lowe NK. The nature of labor pain. Am J Obstet Gynecol 2002 (Suppl S) $186: 16-24$.

2 Ferreira CHJ, Payno SMA. Eletroestimulação nervosa transcutânea como recurso de alívio da dor no trabalho de parto. Femina 2002; 30(2):83-6.

3 Mathias RS, Torres ML. Analgesia e anestesia: técnicas em obstetrícia. In: Neme B. Obstetrícia Básica. $2^{\text {a }}$ ed. São Paulo(SP): Savier; 2000. p. 1035-66.

4 Beleza ACS, Nakano AMS, Santos CB. Práticas obstétricas: fatores de risco para o trauma perineal no parto. Femina 2004; 32 (7): 605-10.

5 Robinson JN, Norwitz ER, Cohen AP, Mcelrath TF, Lieberman ES. Epidural analgesia and third or fourth degree laceration in nulliparas. Obstet Gynecol 1999; 94(2): 257-62.

6 Samuelsson E, Ladfords L, Laindblom BG, Hagberg HA. Prospective observational study on tears during vaginal delivery: occurrences and risk factors. Acta Obstet Gynecol Scandinavica 2002; 81: 44-9.

7 Klein MC, Jannssen PA, Macwillian L, Kaczorowski J, Johson B. Determinants of vaginal-perineal integrity and pelvic floor functioning in childbirth. Am J Obstet Gynecol 1997; 176: 403-10.

8 Johanson R. Perineal massage for prevention of perineal trauma in childbirth. Lancet. 2000; 335 (22): 250-51.

9 Steen M, Cooper K, Marchant P, Griffiths-Jones M, Walker JA. Randomised controlled trial to compare the effectiveness of icepacks and epifoam with cooling maternity gel pads at alleviating postnatal perineal trauma. Midwifery 2000; 16: 48-55. 
Analgesia e trauma perineal no parto normal

Monteiro JCS, Pitangui ACR, Sousa L, Beleza ACS, Nakano AMS, Gomes FA Esc Anna Nery Rev Enferm 2009 jan-mar; 13 (1): 140 -144

10 Carrol TG, Engelken M, Mosier MC, Nazir N. Epidural analgesia and severe perineal laceration in a community-based obstetric practice. J Am Board Fam Pract 2003; 16(1): 1-6.

11 Bodner-Adler B, Kimberger 0, Wagenbibichler P et al. The effect of epidural analgesia on the occurrence of obstetric laceration and on the neonatal outcome during spontaneous vaginal delivery. Arch Gynecol Obstet 2002; 267 (2): 81-4.
12 Albers LL, Anderson D, Gracin L, Daniels SM, Hunter C, Sedler KD. Factors related to perineal in childbirth. J Nurs Midwifery 1996; 41(4): 269-76.

13 Carroli G, Belizan J. Episiotomy for vaginal birth. The Cochrane Library 2003; 3. Oxford (USA): Update Software. Cochrane Review. 